\title{
Erratum
}

\section{Erratum: Tong et al., Age-Dependent Rescue by Simvastatin of Alzheimer's Disease Cerebrovascular and Memory Deficits}

In the article "Age-Dependent Rescue by Simvastatin of Alzheimer's Disease Cerebrovascular and Memory Deficits" by Xin-Kang Tong, Clotilde Lecrux, and Edith Hamel, which appeared on pages 4705-4715 of the April 4, 2012 issue, Pedro Rosa-Neto was accidentally left off the author list. The corrected author list is as follows: "Xin-Kang Tong, ${ }^{1}$ Clotilde Lecrux, ${ }^{1}$ Pedro Rosa-Neto, ${ }^{2}$ and Edith Hamel, ${ }^{1}$ " along with his affiliation: ${ }^{2}$ Translational Neuroimaging Laboratory, McGill Centre for Studies in Aging, Douglas Hospital Research Centre. Also, the Author Contributions footnote should have read "Author contributions: X.-K.T. and E.H. designed research; X.-K.T. and C.L. performed research; X.-K.T., C.L., and P. R.-N. analyzed data; X.-K.T. and E.H. wrote the paper.”

DOI: 10.1523/JNEUROSCI.1845-12.2012 